\title{
Electrospun Conjugated Polymer/Fullerene Hybrid Fibers: Photoactive Blends, Conductivity through Tunneling-AFM, Light Scattering, and Perspective for Their Use in Bulk-Heterojunction Organic Solar Cells
}

Zhenhua Yang, ${ }^{\dagger \neq}$ Maria Moffa, ${ }^{\ddagger}$, Ying Liu, $^{\dagger}$ Hongfei Li, ${ }^{\dagger}{ }^{\dagger}$ Luana Persano, ${ }^{\S}$ Andrea Camposeo, ${ }^{\S}$ Rosalba Saija, ${ }^{\prime}$ Maria Antonia Iatì, ${ }^{\perp}$ Onofrio M. Maragò, ${ }^{\perp}$ Dario Pisignano, ${ }^{*}, \AA, \#, \nabla \odot$ Chang-Yong Nam, ${ }^{\circ}$ Eyal Zussman, and Miriam Rafailovich ${ }^{*}{ }^{\dagger}$

${ }^{\dagger}$ Department of Materials Science and Engineering, State University of New York at Stony Brook, Stony Brook, New York 11794-2275, United States

${ }^{\S}$ NEST, Istituto Nanoscienze-CNR, Piazza S. Silvestro 12, I-56127 Pisa, Italy

"Dipartimento di Scienze Matematiche e Informatiche, Scienze Fisiche e Scienze della Terra, Università di Messina, viale F. Stagno D’Alcontres 31, I-98166 Messina, Italy

${ }^{\perp}$ CNR-IPCF, Istituto per i Processi Chimico-Fisici, viale F. Stagno D’Alcontres 37, I-98166 Messina, Italy

${ }^{\#}$ Dipartimento di Matematica e Fisica “Ennio De Giorgi”, Università del Salento, via Arnesano, I-73100 Lecce, Italy

${ }^{\circ}$ Center for Functional Nanomaterials, Brookhaven National Laboratory, Upton, New York 11973-5000, United States

Department of Mechanical Engineering, Technion-Israel Institute of Technology, Haifa 32000, Israel

\section{Supporting Information}

\begin{abstract}
Hybrid conjugated polymer/fullerene filaments based on MEH-PPV/PVP/PCBM were prepared by electrospinning, and their properties were assessed by scanning electron, atomic and lateral-force, tunneling, and confocal microscopies, as well as by attenuated-total-reflection Fourier transform infrared spectroscopy, photoluminescence quantum yield, and spatially resolved fluorescence. Highlighted features include the ribbon shape of the realized fibers and the persistence of a network serving as a template for heterogeneous active layers in solar cell devices. A set of favorable characteristics is evidenced in this way in terms of homogeneous charge-transport behavior and formation of effective interfaces for diffusion and dissociation of photogenerated excitons. The interaction of the organic filaments with light, exhibiting specific light-scattering properties of the nanofibrous mat, might also contribute to spreading incident radiation across the active layers, thus potentially enhancing photovoltaic performance. This method might be applied to other electron donorelectron acceptor material systems for the fabrication of solar cell devices enhanced by nanofibrillar morphologies embedding conjugated polymers and fullerene compounds.

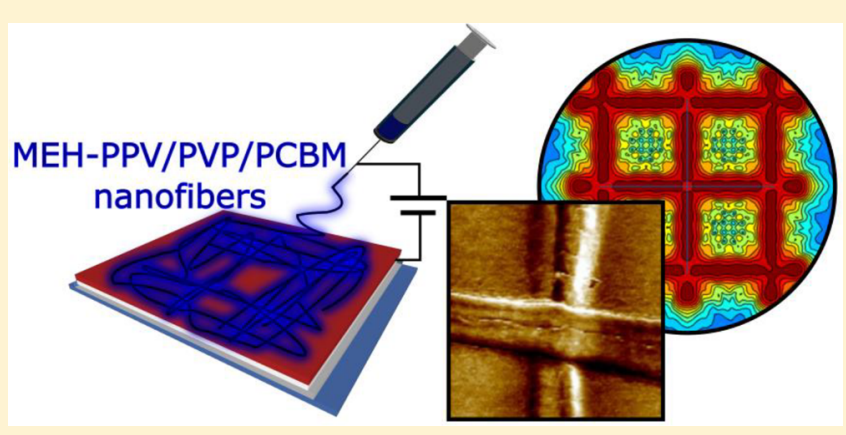

\section{INTRODUCTION}

Bulk-heterojunction (BHJ) polymer solar cells are a field of intense interest because of their flexibility, low cost, and ease of processing. ${ }^{1-4}$ The performance of these devices is known to be highly dependent on the morphology of the active layers ${ }^{5-9}$ and to suffer critically from unfavorable features such as isolated domains or dead ends. ${ }^{4}$ Therefore, a variety of methods have been explored to finely control this morphology, such as thermal annealing, ${ }^{8,10-12}$ solvent annealing, ${ }^{13,14}$ self-assembly of columnar polymer phases, ${ }^{15}$ and micropatterning. ${ }^{16}$ Recently, a different route was developed based on the use of organic nanofibers to optimize the morphology of the donor-acceptor interface. Polymer nanorods ${ }^{12}$ and nanofibers, ${ }^{8}$ prepared by melt-assisted wetting ${ }^{12}$ or by a variety of thermally assisted processing techniques, ${ }^{8,17-19}$ have been shown to increase hole mobility, inducing higher mesoscopic order and crystallinity and enhanced donor-acceptor interfaces and ultimately leading to better device performance compared with that obtained using polymer films, because of the resulting nanoscale and possibly interpenetrating network of fibrillar components. Systems investigated so far have mostly been limited to melts or self-assembled nanostructures made of polythiophene

Received: November 12, 2017

Revised: December 23, 2017

Published: January 25, 2018 
donors, such as core-shell nanorods or layers embedding fibrils of poly(3-hexylthiophene) or poly(3-butylthiophene) and the acceptor phenyl- $\mathrm{C}_{61}$-butyric acid methyl ester (PCBM). ${ }^{8,12,18-20}$ Developing processing methods that can extend the range of conjugated polymers used and offer higher throughput is therefore strongly desirable, in view of the exploitation of networked fibrillar morphologies to enhance the performance of $\mathrm{BHJ}$ solar cells at a larger scale.

Electrospinning is a straightforward technology for the realization of continuous fibers with submicrometer diameters through the application of a high-voltage bias to polymer solutions. $^{21,22}$ This process enables the production of nonwoven materials made of fibers with high surface-area-tovolume ratios that can be deposited in random networks or in uniaxially aligned arrays with three-dimensional porosity. Because of its versatility in terms of usable polymers and blends and its good throughput, electrospinning shows high potential as a method for tailoring the microstructures and compositions of active materials for polymer optoelectronic devices, including solar cells, in a controlled way. ${ }^{23-25}$ Demonstrated applications include field-effect transistors ${ }^{26,27}$ and luministors, ${ }^{28}$ rechargeable batteries, ${ }^{29}$ energy harvesters, ${ }^{30,31}$ light-emitting devices, ${ }^{32-34}$ and nanopatterned lasers. ${ }^{35}$ Electrospun photovoltaic materials could ultimately lead to the development of so-called solar cloths ${ }^{36}$ for smart textile technologies. In addition, organic fibers with diameters matching the wavelength of solar light can exhibit exceptional light-scattering performances, as recently found in insect scales formed by interconnected filaments of chitin, ${ }^{37}$ thus possibly enhancing the coupling of light into the absorbing regions of layered devices. Unfortunately, the electrospinning of conjugated polymers is frequently difficult because of the generally poor viscoelastic behavior of the solutions, which corresponds to the low amounts of molecular entanglements and the limited solubilities of these compounds. Hence, the use of electrospinning methods to produce fibrillar components in $\mathrm{BHJ}$ polymer solar cells and modules has been attempted only rarely. ${ }^{38-42}$ Recently, it was shown that electrospinning can be carried out with a few photoactive polymers, by encasing them in an inert/insulating polymer in a core-shell fiber configuration. $^{39,41}$ This method, however, also proved to be difficult because the shell polymer must first be removed for the device to function, and this step can potentially damage the surface of the remaining functional fibers. Much wider efforts have instead been directed toward the realization of dyesensitized solar cells exploiting electrospun materials. A recent review of such efforts can be found in ref 43 .

Here, we focus on polymer blends to facilitate the electrospinning process. We previously demonstrated that the addition of a secondary polymer component is beneficial for maintaining the structure of films for $\mathrm{BHJ}$ solar cells, even if the second component is not photoactive. ${ }^{15}$ In this work, we report on electrospun poly[2-methoxy-5-(2-ethylhexyloxy)-1,4 phenylenevinylene]/poly(vinylpyrrolidone)/PCBM (MEH-PPV/ PVP/PCBM) blend fibers that are stable, easily produced, and yet photoactive and that significantly improve the performance of $\mathrm{BHJ}$ solar cells. Fibers are incorporated into the device active layer upon deposition of a fully solubilized poly(3-hexylthiophene-2,5-diyl)/PCBM (P3HT/PCBM) backfill layer ${ }^{39}$ on top. Partial redissolution of the electrospun fibers occurs upon deposition of the backfill layer; however, the structural coherence and shape of the fibers are largely maintained in solution-processed multilayer device architec- tures. ${ }^{39}$ The optical and conduction properties of the threedimensional entangled fiber networks are studied by complementary spectroscopies, as well as lateral-force and tunneling atomic force microscopy (AFM), highlighting the formation of compound interfaces driving exciton dissociation in the organic filaments, together with homogeneous charge transport and the generation of streaks along the longitudinal axis of the fibers, primed by PCBM inclusions. The light scattering of these networks is also investigated to demonstrate their capability to direct photons across the mat plane, thus potentially enhancing photoabsorption from the UV to the infrared ranges. The network of organic fibers, serving as a template for P3HT/ PCBM deposition, would also be promising in view of the incorporation of hybrid electrospun conjugated polymer/ fullerene blends into organic photovoltaic devices.

\section{EXPERIMENTAL SECTION}

Materials. MEH-PPV $\left(M_{\mathrm{w}}=150-250 \mathrm{kDa}\right)$ was purchased from Aldrich. PCBM was provided by SES Research, and PVP $\left(M_{\mathrm{w}}=1300 \mathrm{kDa}\right)$ was provided by Alfa Aesar. Chlorobenzene, chloroform, and P3HT $\left(M_{\mathrm{w}}=54-75 \mathrm{kDa}\right)$ were obtained from Sigma-Aldrich. All materials were used without further purification.

Fabrication and Characterization of the Photoactive Blend Nanofibers. The solution for electrospinning was made of MEH-PPV, PVP, and PCBM dissolved in chloroform at concentrations of 15,15 , and $10 \mathrm{mg} / \mathrm{mL}$, respectively. It was then stirred at room temperature for $12 \mathrm{~h}$ to allow for complete polymer dissolution, loaded in a syringe with a 21-gauge stainless steel needle, and injected through the needle at a constant flow rate $(0.5 \mathrm{~mL} / \mathrm{h}$ ) by a syringe pump (Harvard Apparatus, Holliston, MA). A voltage of $8 \mathrm{kV}$ was applied at the needle using a high-voltage power supply (EL60R0.6-22, Glassman High Voltage, High Bridge, NJ). Electrospinning was carried out at ambient atmosphere, and fibers were collected on square $\left(1.5 \times 1.5 \mathrm{~cm}^{2}\right)$ polymer $/ \mathrm{TiO}_{2} /$ indium tin oxide (ITO)/glass substrates mounted on a rotating disk collector $(4000 \mathrm{rpm})$ at a distance of $15 \mathrm{~cm}$ from the needle. A cross-bar pattern of fibers was obtained by depositing fibers along two mutually perpendicular directions, rotating the substrates by $90^{\circ}$ after the first deposition stage, as inspected by scanning electron microscopy (SEM, FEI, Hillsboro, OR). Prior to electrospinning, the substrates were carefully prepared as described in the next section.

UV-visible absorption spectra were obtained on a Thermo Scientific Evolution $200 \mathrm{UV}-$ vis spectrophotometer. The photoluminescence (PL) properties of the fibers were examined in micro-PL mode using a confocal microscopy system composed of an inverted microscope (Eclipse Ti, Nikon) and a laser scanning head (A1R MP, Nikon). Toward this aim, samples were excited with an $\operatorname{Ar}^{+}$laser $\left(\lambda_{\text {exc }}=488 \mathrm{~nm}\right)$ through a $20 \times$ objective (numerical aperture $=0.5$ ), whereas the intensity of the fluorescence, collected by the same excitation objective, was measured using a spectral detection unit equipped with a multianode photomultiplier (Nikon). This allowed spatially resolved spectra and fluorescence images to be collected.

PL quantum yield measurements were performed following the procedure reported in ref 44 . Fiber samples deposited on quartz substrates were positioned in an integrating sphere and excited with a UV light-emitting diode (peak emission wavelength $=300 \mathrm{~nm}$, line width $=18 \mathrm{~nm}$ ). The excitation and emission optical signals were collected by an optical fiber 
coupled to a monochromator (iHR320, Jobin Yvon) and measured by a charge-coupled-device camera (Symphony, Jobin Yvon). Attenuated-total-reflectance Fourier transform infrared (FTIR) spectroscopy was performed on electrospun fibers using a Spectrum 100 spectrometer (PerkinElmer, Waltham, MA) equipped with a $\mathrm{ZnSe}$ crystal for coupling (PerkinElmer). Images of the surface topography and electrical conductivity were also obtained using a Bruker Dimension Icon atomic force microscope (Multimode, Bruker) operating in PeakForce tunneling mode.

Devices. ITO-coated glass was polished in UV/ozone for 10 min to remove any organic impurities. $\mathrm{A} \mathrm{TiO}_{2}$ solution was prepared according to previous reports. ${ }^{45}$ Briefly, the solution was obtained by dissolving $1 \mathrm{~mL}$ of $\mathrm{Ti}\left(\mathrm{OC}_{4} \mathrm{H}_{9}\right)_{4}$ in $10 \mathrm{~mL}$ of ethanol and then adding $1 \mathrm{~mL}$ of $\mathrm{CH}_{3} \mathrm{COOH}$, followed by 1 $\mathrm{mL}$ of acetylacetone and $1 \mathrm{~mL}$ of deionized water. The solution was stirred at room temperature for $30 \mathrm{~min}$ before each reagent was added. A 30-nm-thick $\mathrm{TiO}_{2}$ layer was then spun onto the ITO at $3000 \mathrm{rpm}$ for $20 \mathrm{~s}$ and baked in air at $400{ }^{\circ} \mathrm{C}$ for $2 \mathrm{~h}$ on a hot plate. To maximize adhesion of the fibers, a $\sim 10$-nm-thick film of MEH-PPV/PVP/PCBM was spin-cast on the $\mathrm{TiO}_{2}$ layer at $6000 \mathrm{rpm}$ for $30 \mathrm{~s}$. MEH-PPV/PVP/PCBM fibers were then electrospun as described above. A solution of P3HT and PCBM ( 15 and $9 \mathrm{mg} / \mathrm{mL}$, respectively, in chlorobenzene) was used to interconnect the fibers upon spin-coating at $1000 \mathrm{rpm}$ for $30 \mathrm{~s}$. Samples were then annealed at $150{ }^{\circ} \mathrm{C}$ for $10 \mathrm{~min}$ in a vacuum oven. The resulting thickness of the active layer was $(121 \pm 7)$ $\mathrm{nm}$. Finally, the devices were completed by thermal evaporation of 8-nm $\mathrm{MoO}_{3}$ and 100-nm Ag electrodes with a Kurt J. Lesker PVD 75 vacuum deposition system at Brookhaven National Laboratory. Control devices were prepared according to the same procedure but without electrospun fibers; their thickness was $(112 \pm 5) \mathrm{nm}$. The performance of the thus-obtained solar cells was tested using a $150 \mathrm{~W}$ solar simulator (Oriel) with an AM 1.5G filter for solar illumination. The light intensity was adjusted to $100 \mathrm{~mW} \mathrm{~cm}^{-2}$ with a calibrated thermopile detector (Oriel).

Light Scattering at Nanofibers. Light-scattering calculations for the developed material were carried out in the Tmatrix formalism. We considered the fiber structure to be composed of aggregates of spheres embedded in a homogeneous, isotropic, indefinite medium. The optical properties of the subunit spheres and the surrounding medium were calculated using the Bruggeman description ${ }^{46}$ (see Supporting Information for details). The incident field was a polarized plane wave (whose scattering results were eventually averaged over the in-plane polarization angle); hence, the total field outside the particle was the sum of the incident and scattered fields. The scattered field was obtained by applying the boundary conditions across the surface of each particle in the structure, linking the internal and external fields. The scattering problem was solved by the T-matrix method, ${ }^{47-52}$ based on the definition of a linear operator relating the incident field to the scattered field. ${ }^{47}$ In brief, the starting point of the method was the field expansion in terms of the spherical multipole fields, that is, the vector solutions of the Maxwell equations in a homogeneous medium that were simultaneous eigenfunctions of the angular momentum and the parity operators. The operator $S$, called the transition operator, was introduced thanks to the linearity of the Maxwell equations and the equations expressing the boundary conditions across the surface of the particle. The representation of the operator $S$ on the basis of the spherical multipole fields gave the T-matrix
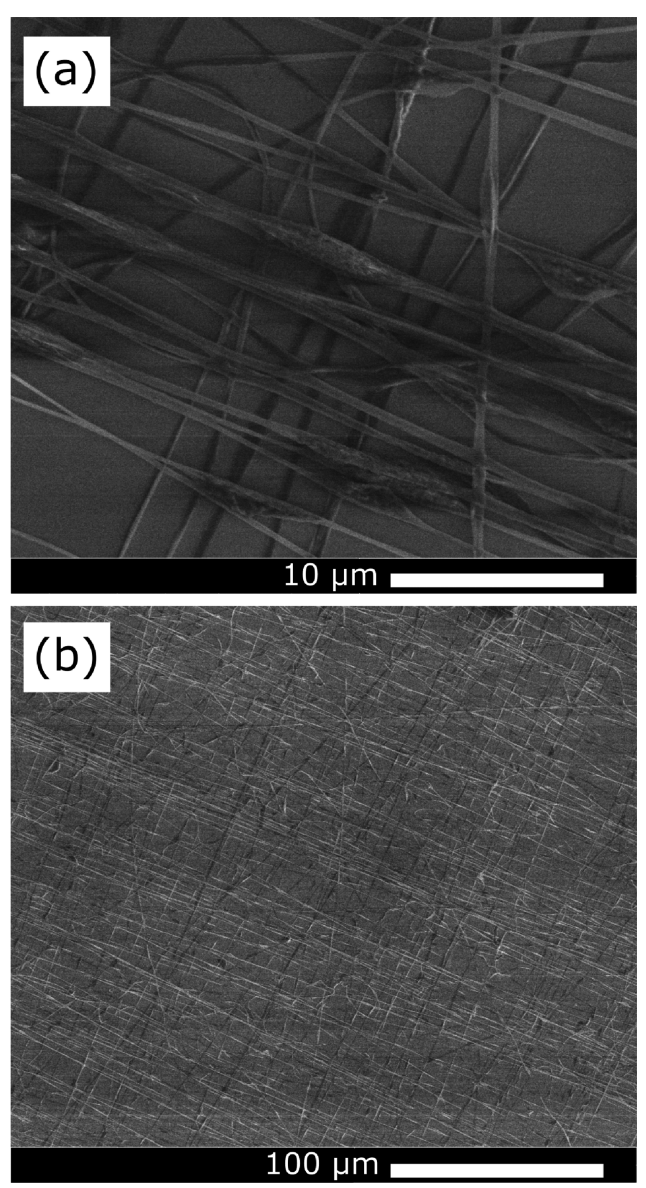

Figure 1. SEM micrographs of electrospun MEH-PPV/PVP/PCBM fibers in cross-bar configuration, imaged at different magnifications. A locally rough surface, suggesting that phase separation occurs in the blend during electrospinning, is visible in the thicker regions in the fibers in panel a. Images also show that fibers are superimposed at different heights on the substrate, thus generating a truly threedimensional network.

whose elements, $S_{l m l^{\prime} m^{\prime}}{ }^{\left(p p^{\prime}\right)}$, (where $p$ is the parity index and $l$ and $m$ are angular momentum indices), contained all of the information about the scattering process but were independent of the state of polarization of the incident field. Here, we used the cluster model ${ }^{48-50}$ to get the scattering properties of fibrous mats embedded in a surrounding medium. The cluster model is a special case because the T-matrix approach solves the scattering problem by an aggregate (cluster) of spheres without resorting to any approximation. ${ }^{50}$ This is highly useful because it allows for the simulation of many situations of practical interest involving nonspherical scatterers. We also highlight that, to calculate the T-matrix of a cluster, one must solve a linear system of equations with, in principle, infinite order. Thus, the system must be truncated to some finite order by including in the multipole expansions terms up to this truncation, chosen so to ensure the convergence of the calculations. As a consequence, the computational demand for these calculations increases with the cube of the number of spheres. $^{51-53}$

\section{RESULTS AND DISCUSSION}

In our samples, a backfill layer of fully solubilized P3HT/ PCBM was spin-cast onto the top of the nanofibrous layer, thus interconnecting the electrospun fibers and avoiding short- 

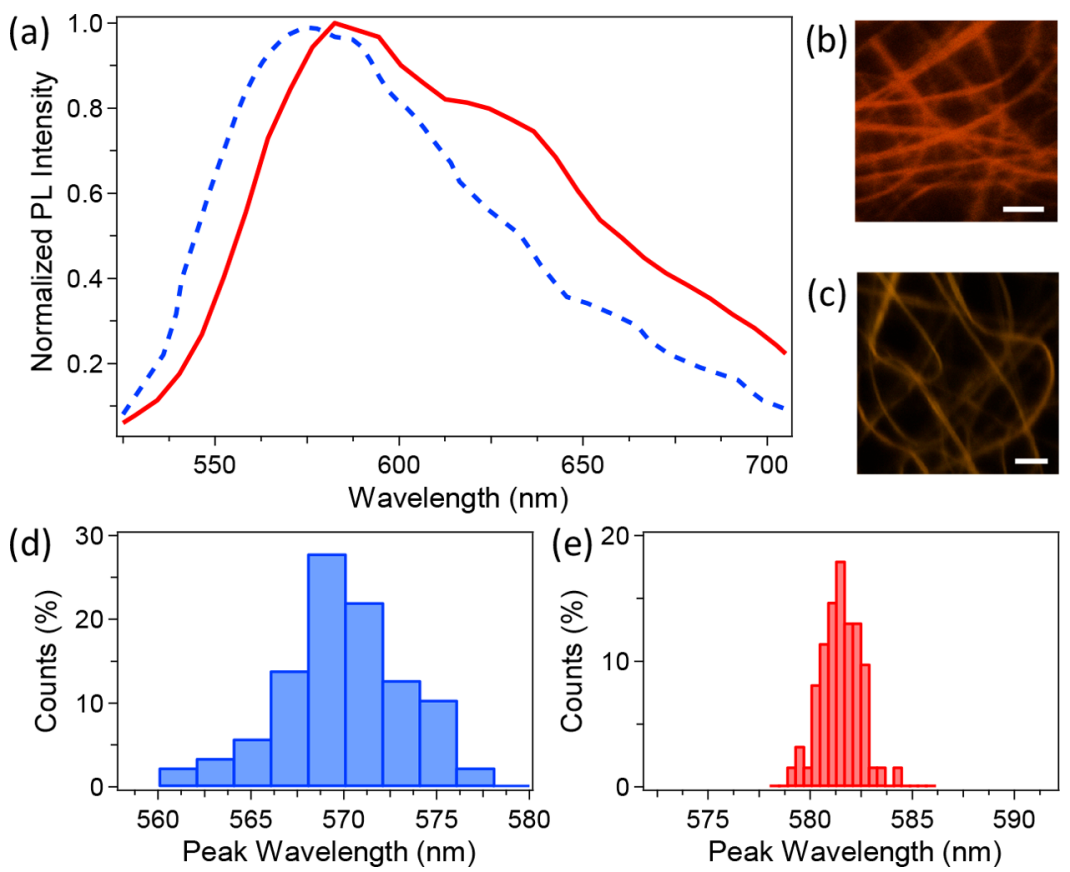

Figure 2. (a) PL spectra of MEH-PPV/PVP nanofibers (red continuous line) and of MEH-PPV/PVP/PCBM nanofibers (blue dashed line). (b,c) Exemplary fluorescence maps of (b) MEH-PPV/PVP and (c) MEH-PPV/PVP/PCBM measured by confocal microscopy. Scale bars $=10 \mu \mathrm{m}$. (d,e) Distributions of the peak emission wavelengths for (d) MEH-PPV/PVP/PCBM and (e) MEH-PPV/PVP nanofibers, obtained after measuring spatially resolved fluorescence spectra. The spectra used for the analysis were averaged over a $\sim 1 \mu \mathrm{m}^{2}$ area along the length of the fibers.

circuit contacts of sandwiching electrodes, which would be likely to occur because of the highly porous nature of the network of filaments. P3HT/PCBM as a backfill layer has very good film-forming properties, and through two different types of photoactive polymers (i.e., MEH-PPV and P3HT), the resulting devices would be able to increase their acceptance range of incoming light frequencies. Finally, $\mathrm{MoO}_{3}$ and $\mathrm{Ag}$ were deposited as an electron-blocking layer and the anode, respectively. For the deposition of the nanofibrous layers, electrospinning was optimized to provide a three-dimensional and interconnected structure made of MEH-PPV fibers blended with PCBM. To overcome the poor spinnability of pristine MEH-PPV, because of its limited molecular weight (150-250 $\mathrm{kDa}$ ) and generally poor viscoelastic behavior in solution, the easily spinnable component of PVP was added to the solution. MEH-PPV and PVP were blended at a 1:1 (w/w) relative concentration, which was found to lead to fiber formation with stable electrified jets and good efficiency. In addition, we tuned the density of the nanofibrous network to obtain a given degree of coverage onto the surface. A deposition time of a few minutes was used to obtain thicknesses ranging from one to a few superimposed layers of fibers. Unlike in previous works, ${ }^{39,41}$ we did not employ sacrificial sheaths in core-shell nanofibers, which would need additional processing steps to be removed, but instead used a unique blend system relying on percolative paths for internal charge transport. The morphology of the $\mathrm{MEH}-\mathrm{PPV} / \mathrm{PVP} / \mathrm{PCBM}$ electrospun fibers, shown in Figure 1, highlights that three-dimensional nonwoven networks were formed in which the fibers were uniformly dispersed and exhibited a ribbon shape and a transversal size $\sim 300-450 \mathrm{~nm}$, with very rare beads over a large area. The domains observed on the fiber surface suggest phase separation of the different polymer components at scales (few tens of nanometers) that match well with the typical exciton diffusion lengths in organics. ${ }^{4}$
FTIR spectra of the electrospun MEH-PPV/PVP/PCBM fibers show a band peaked at $528 \mathrm{~cm}^{-1}$, which is characteristic for the fullerene derivatives ${ }^{54,55}$ (Figure $S 1$ in the Supporting Information). More importantly, the presence of PCBM in these fibers and the formation of effective internal interfaces between the acceptor and the donor compounds in the organic filaments are supported by the reduction of the PL quantum yield found for fibers with fullerene compared to MEH-PPV/ PVP fibers. Indeed, the PL quantum yield of the MEH-PPV/ PVP fibers, measured with an integrating sphere and accounting for the number of emitted photons per incident photon, ${ }^{44}$ is $(11 \pm 1) \%$, decreasing by at least 1 order of magnitude $(<1 \%)$ upon fullerene addition. Furthermore, the PL spectrum of the MEH-PPV/PVP/PCBM fibers is blue-shifted by $10 \mathrm{~nm}$, compared to that of MEH-PPV/PVP fibers (Figure 2a). Such a blue shift also appears in the spatially resolved fluorescence spectra of the fibers measured by micro-PL, allowing for the collection of PL maps with submicrometer spatial resolution (Figure $2 b, c)$. The uniform brightness along the fibers in the micrographs in Figure $2 b, c$ clearly indicates the homogeneous incorporation of MEH-PPV in the electrospun filaments. In particular, the analysis of the spectra collected from different areas of the fiber samples indicates a substantial blue shift of the average peak wavelength (by $12 \mathrm{~nm}$, Figure $2 \mathrm{~d}, \mathrm{e}$ ) because of the presence of PCBM, together with a broadening of the peak wavelength distribution, featuring an increase of the full width at half-maximum from 2 to $7 \mathrm{~nm}$ upon PCBM addition. Following photoexcitation, the emission properties of conjugated polymers, which are multichromophore systems composed of many active subunits, are determined by energy migration, funneling the excitation toward those chromophores that have lower characteristic energies and that emit light. ${ }^{56}$ In pristine electrospun nanofibers made of conjugated polymers, this process occurs on picosecond time scales, can be tailored by the degree of aggregation of the conjugated polymer chains, 
and determines the emission to occur from the more extended and conjugated subunits. ${ }^{57}$ The presence of PBCM and the formation of effective interfaces for the dissociation of photogenerated excitons through electron transfer ${ }^{58}$ lowers the PL quantum yield. In addition, the blue-shifted emission indicates a decreased aggregation of the conjugated polymer chains in composite samples. ${ }^{57,59}$ Overall, these findings support the occurrence of diffusion and dissociation of photogenerated excitons at the interfaces formed within the electrospun nanofibers.

The morphology of the active layer surface resulting from the fiber deposition was studied with lateral-force microscopy and AFM (Figure 3). It can be seen from Figure 3a that the structural coherence of the $\mathrm{MEH}-\mathrm{PPV} / \mathrm{PVP} / \mathrm{PCBM}$ fibers is well maintained after the deposition of the P3HT/PCBM backfill layer. Although with flattening due to partial dissolution, the fiber network still serves as a template ${ }^{39}$ for the active layer (Figure $3 \mathrm{~b}$ ). In this way, a complex, doubleheterojunction structure is maintained in the active film, with
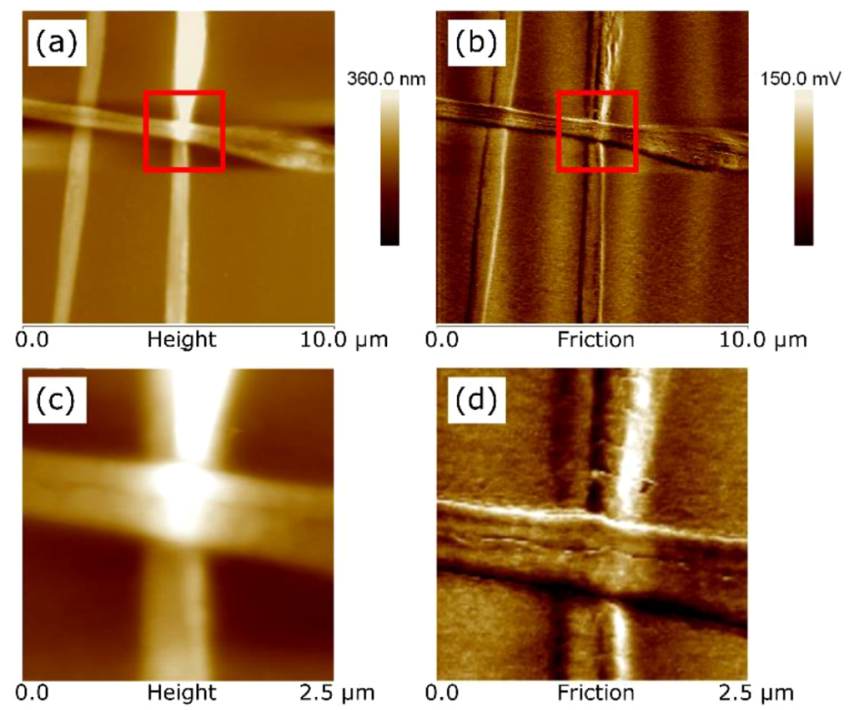

(e)
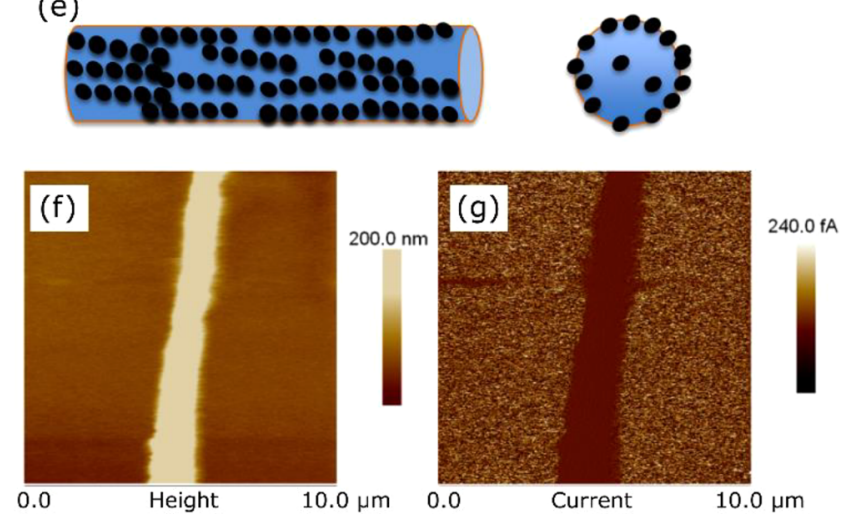

Figure 3. (a) AFM and (b) lateral-force microscopy images $(10 \mu \mathrm{m} \times$ $10 \mu \mathrm{m})$ of cross-bar MEH-PPV/PVP/PCBM fibers deposited on the blend film with addition of a backfill layer. (c,d) Magnified images of the regions delineated by the red squares in panels a and b. (e) Schematic representation of PCBM assembly at the fiber surface. The dark dots represent PCBM nanoparticles (features not to scale compared to the fiber diameter). (f,g) TUNA images $(10 \mu \mathrm{m} \times 10$ $\mu \mathrm{m})$ of ITO-glass/ $\mathrm{TiO}_{2} / \mathrm{MEH}$-PPV/PVP/PCBM film/electrospun MEH-PPV/PVP/PCBM fiber samples. (f) Fiber topography and (g) corresponding current map obtained with an applied voltage of $2 \mathrm{~V}$. tandem features due to the presence of diverse conjugated polymers increasing the range of effective absorption for incoming light. The layered structures create multiple donoracceptor interfaces that are formed in the fibers and in the backfill layer, as well as a PCBM component possibly promoting the development of percolative paths for electrons across regions realized with different blends. In panels $c$ and $d$ of Figure 3, we show magnified views of the regions highlighted by the squares in panels a and $b$, respectively, of Figure 3 . Although not visible in the topographic images because of their small heights $(\sim 2 \mathrm{~nm})$, PCBM inclusions can be clearly seen in the friction image (Figure 3d), where they appear slightly darker or harder than their surroundings. The PCBM is arranged in long streaks that run along the length of the fiber. The PCBM that is visible also lies directly on the surface of the fiber, because friction-mode scans are sensitive only to differences in mechanical or adhesion surface forces. By imaging the electrospinning process using fast X-ray analysis, it was previously shown that particle inclusions self-assemble in long streaks that run along the length of a fiber and delineate the stream lines of the electrospining jet. To maintain lamellar flow in the viscous stream, particle inclusions are also pushed toward the surface of the stream, where the deformation they cause is minimized. ${ }^{60}$ Along the jet, because of the dominant effect of axial stretching accompanied by lateral contraction, the particles tend to be segregated toward the surface of the fibers in the as-spun samples. A cartoon of the PCBM distribution in the electrospun fibers, in both side and top views, is shown in Figure 3e. If the PCBM concentration is sufficiently high, a percolative network forms on the surface of the fiber, which might be very effective in increasing the current of a $\mathrm{BHJ}$ photovoltaic device. We also utilized tunneling AFM (TUNA) to investigate the fiber conductivity. Figure $3 \mathrm{f}$ displays a region of an electrospun MEH-PPV/PVP/PCBM filament deposited on the blend film. The corresponding current map for zero bias highlights no significant difference between the fiber and the surrounding film (Figure S2). As the applied voltage is increased to $2 \mathrm{~V}$, the fiber features are brought back and match well with the topography image (Figure $3 \mathrm{~g}$ ). The current detected from the fiber area (about $1.5 \mathrm{fA}$ ) is lower than that from the film because of the higher serial resistance. Indeed, the ratio of the measured current values for the fiber and the surrounding layer $(\sim 25)$ agrees well with the ratio of the corresponding thicknesses in the two probed areas. Overall, these results indicate a homogeneous charge-transport behavior for the adhesion film and the fibers along their transversal direction, as well as conductivity values $\left[(1.02-1.34) \times 10^{-7} \mathrm{~S} /\right.$ $\mathrm{m}$ ] that are adequate for optoelectronic applications such as solar cells.

The transmission spectra of different samples (MEH-PPV/ PVP/PCBM adhesion film, MEH-PPV/PVP/PCBM film with added backfill layer, and MEH-PPV/PVP/PCBM film with fibers and backfill layer) are shown in Figure 4. MEH-PPV/ PVP/PCBM films exhibit a broad absorption at $430-570 \mathrm{~nm}$ and a maximum absorption at about $490 \mathrm{~nm}$ arising from the $\pi$ conjugated structure. In addition, the presence of PCBM can be deduced because of the related absorption at about $300 \mathrm{~nm}^{61}$

Following the addition of the backfill layer, extra peaks at 517 and $556 \mathrm{~nm}$ and one shoulder at $605 \mathrm{~nm}$ are detected, corresponding to $\pi-\pi^{*}$ transitions from $\mathrm{P} 3 \mathrm{HT},{ }^{61}$ together with a significant decrease in the transmitted intensity. Previous research showed that there is no interaction between P3HT and MEH-PPV in terms of absorption spectra and that the 


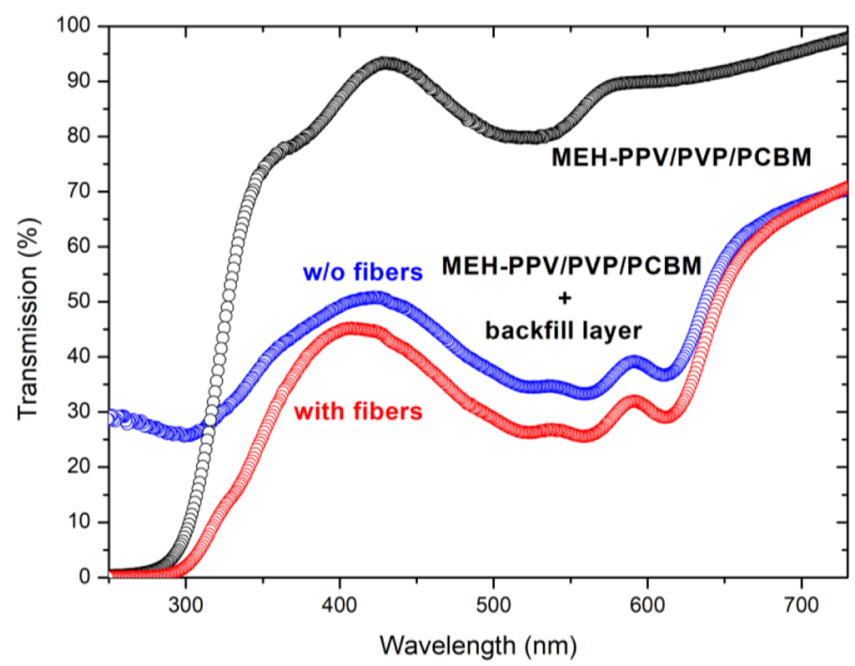

Figure 4. Transmission spectra in the visible and near-infrared regions for a MEH-PPV/PVP/PCBM thin film (black circles), a MEH-PPV/ PVP/PCBM thin film with a backfill layer (blue), and a MEH-PPV/ PVP/PCBM thin film with nanofibers and a backfill layer (red).

absorbed energy of the films of the blend series is independent of the blending ratio. ${ }^{62}$ Therefore, the decrease in transmission through the layer found here can be attributed to the incorporation of the nanofibers.

To elucidate in depth the underlying working mechanisms, the light-scattering properties of the ordered fibers embedded in their external medium were determined by exploiting the transition matrix (T-matrix) formalism. ${ }^{47-49}$ The T-matrix approach combines an accurate description of the scattering process with computational efficiency and a wide range of particle sizes when describing complex nonspherical and composite particles. In this work, we modeled the composite polymer fibers as ordered clusters of spheres, ${ }^{51,52,63}$ as shown in Figure 5a. To mimic the nonspherical fiber section as well as the arrangement of the filaments used in devices, we considered ordered arrays of sphere dimers with diameters of $300 \mathrm{~nm}$ as the fiber short axis and the resulting transverse size of each dimer of $600 \mathrm{~nm}$, corresponding to the transverse fiber size.

The optical constants of the fibers and of the surrounding medium were calculated using an effective-medium theory exploiting the Bruggeman effective dielectric function for composite materials (Supporting Information), ${ }^{47}$ where the different compositions of the fiber and external medium were accurately taken into account. Light-scattering maps for different wavelengths of incident light, matching different regions of the solar spectrum (from the ultraviolet to the nearinfrared), are shown in Figure $5 b-d$. For each wavelength, a map was obtained for the scattered field normalized to an unpolarized incident field intensity, $\left|E_{\mathrm{S}} / E_{0}\right|^{2}$. From these maps, it is clear that the fibrous structures scatter light in an efficient way, producing hot spots of radiation spreading across the involved photovoltaic interfaces. In addition, light scattering is stronger in the near-infrared region, where the average thickness of deposited fibers is closer to the incident wavelength. Moreover, the scattered light at relatively longer wavelengths is spread out over and outside the fibrous structure, thus reliably increasing optical coupling with the surrounding layer and ultimately photon absorption across the organics.

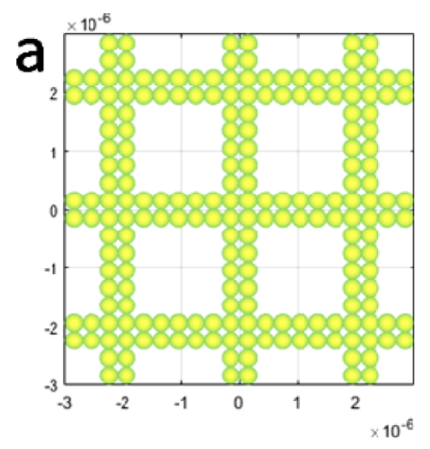

\section{b}
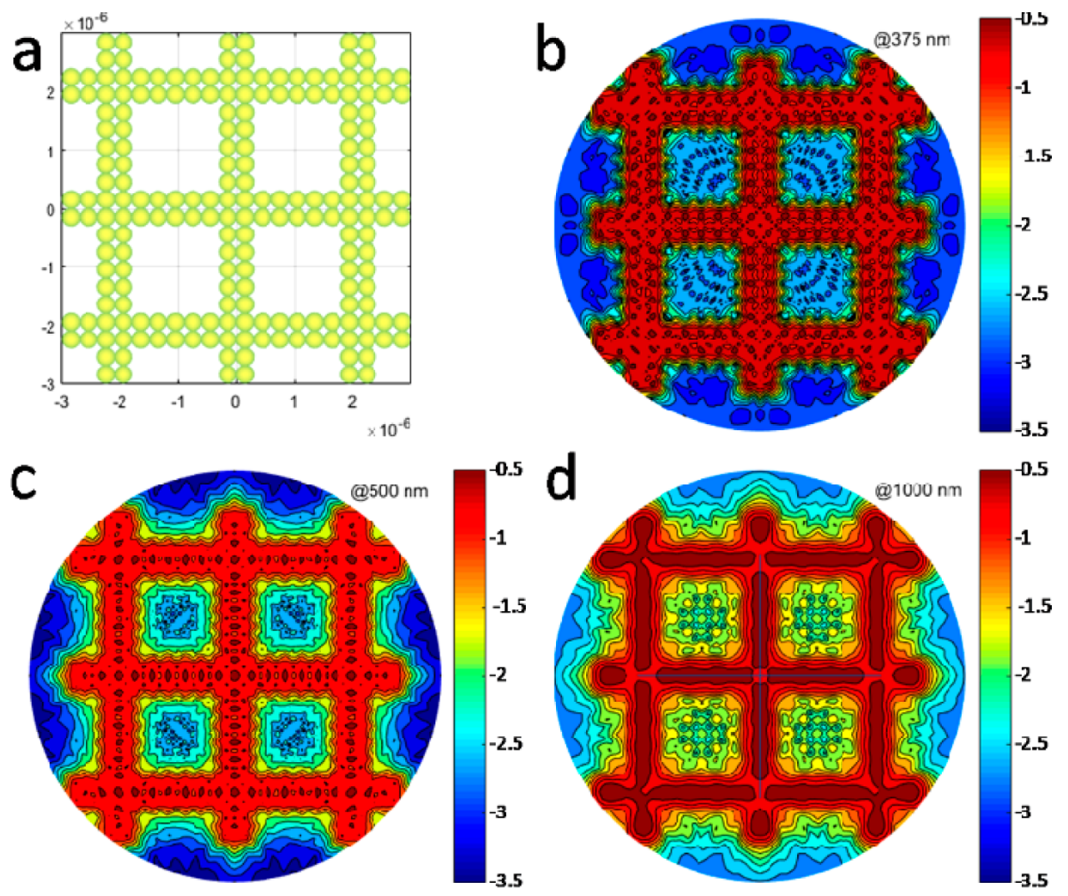

Figure 5. (a) Sketch of the model structure for the light-scattering calculations from the ordered, composite polymer nanofiber mats embedded in a dielectric medium. The organic filaments were modeled as aggregates of spheres with a 300-nm diameter to match the thickness of the ribbonshaped fibers realized in the experiments. The optical properties of the nanofibers and the external medium were obtained using an effective-medium theory (see text). (b-d) Normalized intensity maps $\left(\left|E_{S} / E_{0}\right|^{2}\right.$, on a logarithmic scale) of the scattered field for the fiber mats at different wavelentgths: (b) 375 , (c) 500, and (d) $1000 \mathrm{~nm}$. In all of the calculations, the light was considered to propagate orthogonally to the fiber mats and with a polarization that was averaged in the plane. The fiber structures scattered more strongly in the infrared range because of the better-matching average thickness, with scattered light also being more spread out over the structure. 


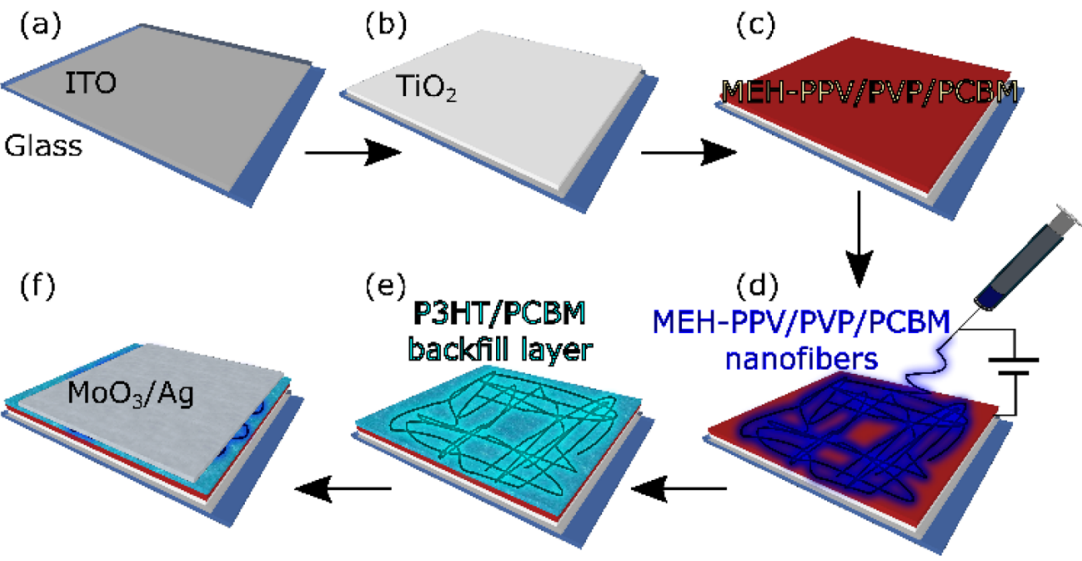

Figure 6. Scheme of the process for realizing solar cell devices based on electrospun MEH-PPV/PVP/PCBM nanofibers. The fibers were spun on (a) ITO/glass substrates, after which (b) a 30-nm-thick $\mathrm{TiO}_{2}$ layer and (c) a spin-cast MEH-PPV/PVP/PCBM film were deposited. After (d) electrospinning, (e) $\mathrm{P} 3 \mathrm{HT} / \mathrm{PCBM}$ was spin-cast to define the template active layer, and (f) the electron-blocking layer and top electrode were thermally evaporated.

The fabrication process of a prototype $\mathrm{BHJ}$ solar cell embedding electrospun MEH-PPV/PVP/PCBM fibers is summarized in Figure 6, with the corresponding current density-voltage curves shown in Figure 7. With the inclusion

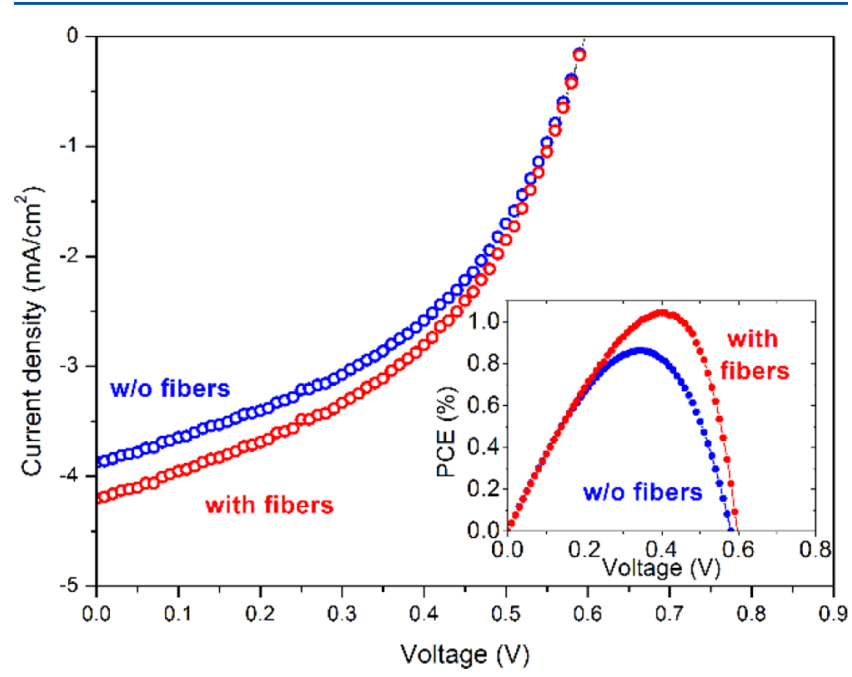

Figure 7. Current density-voltage characteristics of devices with active layers of MEH-PPV/PVP/PCBM film without nanofibers (blue symbols) and with nanofibers (red symbols). Inset: Corresponding PCE curves for exemplary devices without (blue symbols) and with (red symbols) nanofibers.

of electrospun MEH-PPV/PVP/PCBM fibers in the active layer, the $J_{\mathrm{sc}}$ and $\mathrm{FF}$ values increase, on average, by $0.39 \mathrm{~mA}$ $\mathrm{cm}^{-2}$ (i.e., from 3.63 to $4.02 \mathrm{~mA} \mathrm{~cm}{ }^{-2}$ ) and $4.4 \%$ (from $41 \%$ ), respectively, which is indicative of enhanced photon absorption according to the above-reported mechanisms and, consequently, enhanced free-charge generation. ${ }^{64}$ No significant change occurs in the open-circuit voltage $\left(V_{\text {oc }}=0.56-0.57 \mathrm{~V}\right)$, suggesting a negligible effect on shunt resistance. ${ }^{42}$ Finally, the power conversion efficiency (PCE) is increased by about $20 \%$ upon nanofiber embedment (from $\sim 0.8 \%$ to $\geq 1 \%$; see the inset of Figure 7). The performance improvement is attributed to the active layer template formed with electrospun nanofibers, which affects various properties of the overall device, including the internal light-scattering properties as specified above, in turn enhancing the internal absorption of incident photons. In addition, the nanofiber template not only serves as a guide path for electron transport but also increases the interfacial area between donor and acceptor to induce more exciton dissociation. Such a template approach is promising in view of achieving higher efficiencies under ambient conditions with other polymer solar cell systems.

\section{CONCLUSIONS}

In summary, MEH-PPV/PVP/PCBM nanofibers can be successfully prepared by electrospinning and incorporated into the active layer of polymer solar cell devices. The entangled fiber network serves as a template for the active layer, with effects promoting the diffusion and dissociation of photogenerated excitons at the involved organic interfaces, as well as light scattering, redirecting incident photons across active layers. The electrospinning process forces particle inclusions toward the exterior of the spinning stream. As a result, a particle-rich layer is formed with a well-defined pathway for electron conduction, as illustrated in Figure 3e. This conformation could lead to an increased current and fill factor upon inclusion of fiber structures in $\mathrm{BHJ}$ solar cells. So far, the observed performances from these architectures are largely in line with measurements reported for self-assembled polythiophene nanofibers in PCBM. ${ }^{18}$ A body of literature exists on various optimized configurations exploiting blends of P3HT and PCBM, with PCEs of about $3 \%$ on average. ${ }^{65}$ The findings reported herein are relevant in this framework, because they can be easily generalized to other conjugated polymers and because they highlight the synergy of the phase-separation, transport, and light-scattering properties of organic nanostructures embedded in bulk-heterojunction solar cells. Furthermore, various strategies can be developed to further improve along these directions. In particular, electrospun organic nanofibers can be easily doped or decorated with semiconducting quantum dots, ${ }^{66,67}$ which has been found to be an excellent route to enhancing donor-acceptor electronic interactions. ${ }^{68}$ Also, electrospinning nanofibers based on conjugated polymers electrospun in a controlled nitrogen atmosphere might lead to better charge transport because of the reduced incorporation of oxygen during fabrication. ${ }^{69}$ This methodology is likely to be extended to other donor-acceptor material systems and to flexible solar cell devices, as suggested 
by recent evidence on highly stable bendable field-effect transistors. $^{70}$

\section{ASSOCIATED CONTENT}

\section{S Supporting Information}

The Supporting Information is available free of charge on the ACS Publications website at DOI: 10.1021/acs.jpcc.7b11188.

Diameter distributions, further fiber characterization material, and device schemes (PDF)

\section{AUTHOR INFORMATION}

\section{Corresponding Authors}

*E-mail: dario.pisignano@unipi.it.

*E-mail: miriam.rafailovich@stonybrook.edu.

\section{ORCID $\odot$}

Hongfei Li: 0000-0002-0094-6707

Dario Pisignano: 0000-0003-3758-5199

Chang-Yong Nam: 0000-0002-9093-4063

\section{Present Address}

${ }^{\nabla}$ Dipartimento di Fisica, Università di Pisa and CNR Istituto Nanoscienze, Largo B. Pontecorvo 3, I-56127 Pisa, Italy.

\section{Author Contributions}

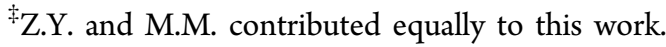

\section{Notes}

The authors declare no competing financial interest.

\section{ACKNOWLEDGMENTS}

The research leading to these results received funding from the European Research Council under the European Union's Seventh Framework Programme (FP/2007-2013)/ERC Grant Agreement 306357 (ERC Starting Grant "NANO-JETS"). M.R. and Z.Y. gratefully acknowledge the support of the National Science Foundation (NSF Grant INSPIRE\#1344267). The Apulia Networks of Public Research Laboratories Wafitech (09) and MITT (13), and the Advanced Energy CenterThINC facility are also acknowledged.

\section{REFERENCES}

(1) Günes, S.; Neugebauer, H.; Sariciftci, N. S. Conjugated PolymerBased Organic Solar Cells. Chem. Rev. 2007, 107, 1324-1338.

(2) Dennler, G.; Scharber, M. C.; Brabec, C. J. Polymer-Fullerene Bulk-Heterojunction Solar Cells. Adv. Mater. 2009, 21, 1323-1338.

(3) Beaujuge, P. M.; Fréchet, J. M. Molecular Design and Ordering Effects in $\pi$-Functional Materials for Transistor and Solar Cell Applications. J. Am. Chem. Soc. 2011, 133, 20009-20029.

(4) Mayer, A. C.; Scully, S. R.; Hardin, B. E.; Rowell, M. W.; McGehee, M. D. Polymer-Based Solar Cells. Mater. Today 2007, 10, $28-33$.

(5) Liu, F.; Gu, Y.; Jung, J. W.; Jo, W. H.; Russell, T. P. On the Morphology of Polymer-Based Photovoltaics. J. Polym. Sci., Part B: Polym. Phys. 2012, 50, 1018-1044.

(6) Chen, W.; Nikiforov, M. P.; Darling, S. B. Morphology Characterization in Organic and Hybrid Solar Cells. Energy Environ. Sci. 2012, 5, 8045-8074.

(7) Moulé, A. J.; Meerholz, K. Morphology Control in SolutionProcessed Bulk-Heterojunction Solar Cell Mixtures. Adv. Funct. Mater. 2009, 19, 3028-3036.

(8) Yang, X.; Loos, J.; Veenstra, S. C.; Verhees, W. J.; Wienk, M. M.; Kroon, J. M.; Michels, M. A.; Janssen, R. A. Nanoscale Morphology of High-Performance Polymer Solar Cells. Nano Lett. 2005, 5, 579-583.

(9) Xin, H.; Reid, O. G.; Ren, G.; Kim, F. S.; Ginger, D. S.; Jenekhe, S. A. Polymer Nanowire/Fullerene Bulk Heterojunction Solar Cells:
How Nanostructure Determines Photovoltaic Properties. ACS Nano 2010, 4, 1861-1872.

(10) Nam, S.; Shin, M.; Kim, H.; Kim, Y. Temperature/TimeDependent Crystallization of Polythiophene:Fullerene Bulk Heterojunction Films for Polymer Solar Cells. Nanoscale 2010, 2, 2384-2389.

(11) Clarke, T. M.; Ballantyne, A. M.; Nelson, J.; Bradley, D. D.; Durrant, J. R. Free Energy Control of Charge Photogeneration in Polythiophene/Fullerene Solar Cells: The Influence of Thermal Annealing on P3HT/PCBM Blends. Adv. Funct. Mater. 2008, 18, 4029-4035.

(12) Wang, H.-S.; Lin, L.-H.; Chen, S.-Y.; Wang, Y.-L.; Wei, K.-H. Ordered Polythiophene/Fullerene Composite Core-Shell Nanorod Arrays for Solar Cell Applications. Nanotechnology 2009, 20, 075201.

(13) Tang, H.; Lu, G.; Li, L.; Li, J.; Wang, Y.; Yang, X. Precise Construction of PCBM Aggregates for Polymer Solar Cells via MultiStep Controlled Solvent Vapor Annealing. J. Mater. Chem. 2010, 20, 683-688.

(14) Jo, J.; Na, S. I.; Kim, S. S.; Lee, T. W.; Chung, Y.; Kang, S. J.; Vak, D.; Kim, D. Y. Three-Dimensional Bulk Heterojunction Morphology for Achieving High Internal Quantum Efficiency in Polymer Solar Cells. Adv. Funct. Mater. 2009, 19, 2398-2406.

(15) Pan, C.; Li, H.; Akgun, B.; Satijia, S. K.; Zhu, Y.; Xu, D.; Ortiz, J.; Gersappe, D.; Rafailovich, M. H. Enhancing the Efficiency of Bulk Heterojunction Solar Cells via Templated Self-Assembly. Macromolecules 2013, 46, 1812-1819.

(16) Chen, F.-C.; Lin, Y.-K.; Ko, C.-J. Submicron-Scale Manipulation of Phase Separation in Organic Solar Cells. Appl. Phys. Lett. 2008, 92, 023307.

(17) Zhao, Y.; Shao, S.; Xie, Z.; Geng, Y.; Wang, L. Effect of Poly (3hexylthiophene) Nanofibrils on Charge Separation and Transport in Polymer Bulk Heterojunction Photovoltaic Cells. J. Phys. Chem. C 2009, 113, 17235-17239.

(18) Xin, H.; Kim, F. S.; Jenekhe, S. A. Highly Efficient Solar Cells Based on Poly(3-butylthiophene) Nanowires. J. Am. Chem. Soc. 2008, $130,5424-5425$.

(19) Xin, H.; Ren, G.; Kim, F. S.; Jenekhe, S. A. Bulk Heterojunction Solar Cells from Poly(3-butylthiophene)/Fullerene Blends: In Situ Self-Assembly of Nanowires, Morphology, Charge Transport, and Photovoltaic Properties. Chem. Mater. 2008, 20, 6199-6207.

(20) Berson, S.; De Bettignies, R.; Bailly, S.; Guillerez, S. Poly(3hexylthiophene) Fibers for Photovoltaic Applications. Adv. Funct. Mater. 2007, 17, 1377-1384.

(21) Reneker, D. H.; Chun, I. Nanometre Diameter Fibres of Polymer, Produced by Electrospinning. Nanotechnology 1996, 7, 216223.

(22) Greiner, A.; Wendorff, J. H. Electrospinning: a Fascinating Method for the Preparation of Ultrathin Fibers. Angew. Chem., Int. Ed. 2007, 46, 5670-5703.

(23) Persano, L.; Camposeo, A.; Pisignano, D. Active Polymer Nanofibers for Photonics, Electronics, Energy Generation and Micromechanics. Prog. Polym. Sci. 2015, 43, 48-95.

(24) Cho, H.; Min, S.-Y.; Lee, T.-W. Electrospun Organic Nanofiber Electronics and Photonics. Macromol. Mater. Eng. 2013, 298, 475-486.

(25) Min, S.-Y.; Kim, T. S.; Lee, Y.; Cho, H.; Xu, W.; Lee, T.-W. Organic Nanowire Fabrication and Device Applications. Small 2015, $11,45-62$.

(26) Babel, A.; Li, D.; Xia, Y.; Jenekhe, S. A. Electrospun Nanofibers of Blends of Conjugated Polymers: Morphology, Optical Properties, and Field-Effect Transistors. Macromolecules 2005, 38, 4705-4711.

(27) Lee, S. W.; Lee, H. J.; Choi, J. H.; Koh, W. G.; Myoung, J. M.; Hur, J. H.; Park, J. J.; Cho, J. H.; Jeong, U. Periodic Array of Polyelectrolyte-Gated Organic Transistors from Electrospun Poly(3hexylthiophene) Nanofibers. Nano Lett. 2010, 10, 347-351.

(28) Tu, D.; Pagliara, S.; Camposeo, A.; Persano, L.; Cingolani, R.; Pisignano, D. Single Light-Emitting Polymer Nanofiber Field-Effect Transistors. Nanoscale 2010, 2, 2217-2222.

(29) Ji, L.; Zhang, X. Fabrication of Porous Carbon Nanofibers and Their Application as Anode Materials for Rechargeable Lithium-Ion Batteries. Nanotechnology 2009, 20, 155705. 
(30) Chang, C.; Tran, V. H.; Wang, J.; Fuh, Y.-K.; Lin, L. DirectWrite Piezoelectric Polymeric Nanogenerator with High Energy Conversion Efficiency. Nano Lett. 2010, 10, 726-731.

(31) Persano, L.; Dagdeviren, C.; Maruccio, C.; De Lorenzis, L.; Pisignano, D. Cooperativity in the Enhanced Piezoelectric Response of Polymer Nanowires. Adv. Mater. 2014, 26, 7574-7580.

(32) Moran-Mirabal, J. M.; Slinker, J. D.; DeFranco, J. A.; Verbridge, S. S.; Ilic, R.; Flores-Torres, S.; Abruña, H.; Malliaras, G. G.; Craighead, H. Electrospun Light-Emitting Nanofibers. Nano Lett. 2007, 7, 458-463.

(33) Vohra, V.; Giovanella, U.; Tubino, R.; Murata, H.; Botta, C. Electroluminescence from Conjugated Polymer Electrospun Nanofibers in Solution Processable Organic Light-Emitting Diodes. ACS Nano 2011, 5, 5572-5578.

(34) Min, S.-Y.; Bang, J.; Park, J.; Lee, C.-L.; Lee, S.; Park, J.-J.; Jeong, U.; Kim, S.; Lee, T.-W. Electrospun Polymer/Quantum Dot Composite Fibers as Down Conversion Phosphor Layers for White Light-Emitting Diodes. RSC Adv. 2014, 4, 11585-11589.

(35) Persano, L.; Camposeo, A.; Del Carro, P.; Fasano, V.; Moffa, M.; Manco, R.; D’Agostino, S.; Pisignano, D. Distributed Feedback Imprinted Electrospun Fiber Lasers. Adv. Mater. 2014, 26, 6542-6547.

(36) Sundarrajan, S.; Murugan, R.; Nair, A. S.; Ramakrishna, S. Fabrication of P3HT/PCBM Solar Cloth by Electrospinning Technique. Mater. Lett. 2010, 64, 2369-2372.

(37) Burresi, M.; Cortese, L.; Pattelli, L.; Kolle, M.; Vukusic, P.; Wiersma, D. S.; Steiner, U.; Vignolini, S. Bright-White Beetle Scales Optimise Multiple Scattering of Light. Sci. Rep. 2015, 4, 6075.

(38) Kim, T.; Yang, S. J.; Kim, S. K.; Choi, H. S.; Park, C. R. Preparation of PCDTBT Nanofibers with a Diameter of $20 \mathrm{~nm}$ and Their Application to Air-Processed Organic Solar Cells. Nanoscale 2014, 6, 2847-2854.

(39) Bedford, N. M.; Dickerson, M. B.; Drummy, L. F.; Koerner, H.; Singh, K. M.; Vasudev, M. C.; Durstock, M. F.; Naik, R. R.; Steckl, A. J. Nanofiber-Based Bulk-Heterojunction Organic Solar Cells Using Coaxial Electrospinning. Adv. Energy. Mater. 2012, 2, 1136-1144.

(40) Kim, T.; Yang, S. J.; Sung, S. J.; Kim, Y. S.; Chang, M. S.; Jung, H.; Park, C. R. Highly Reproducible Thermocontrolled Electrospun Fiber Based Organic Photovoltaic Devices. ACS Appl. Mater. Interfaces 2015, 7, 4481-4487.

(41) Kim, M.; Jo, S. B.; Park, J. H.; Cho, K. Flexible Lateral Organic Solar Cells with Core-Shell Structured Organic Nanofibers. Nano Energy 2015, 18, 97-108.

(42) Khanum, K. K.; Ramamurthy, P. C. Instigating Network Structure in Bulk Heterojunction Organic Solar Cells Creating a Unique Approach in Augmenting the Optical Absorption. Polymer 2016, 91, 146-155.

(43) Joly, D.; Jung, J.-W.; Kim, I.-D.; Demadrille, R. Electrospun Materials for Solar Energy Conversion: Innovations and Trends. J. Mater. Chem. C 2016, 4, 10173-10197.

(44) Greenham, N. C.; Samuel, I. D. W.; Hayes, G. R.; Phillips, R. T.; Kessener, Y. A. R. R.; Moratti, S. C.; Holmes, A. B.; Friend, R. H. Measurement of Absolute Photoluminescence Quantum Efficiencies in Conjugated Polymers. Chem. Phys. Lett. 1995, 241, 89-96.

(45) Xue, H.; Kong, X.; Liu, Z.; Liu, C.; Zhou, J.; Chen, W.; Ruan, S.; $\mathrm{Xu}, \mathrm{Q} . \mathrm{TiO}_{2}$ Based Metal-Semiconductor-Metal Ultraviolet Photodetectors. Appl. Phys. Lett. 2007, 90, 201118.

(46) Chylek, P.; Srivastava, V. Dielectric Constant of a Composite Inhomogeneous Medium. Phys. Rev. B: Condens. Matter Mater. Phys. 1983, 27, 5098-5106.

(47) Borghese, F.; Denti, P.; Saija, R. Scattering from Model Nonspherical Particles, 2nd ed.; Springer-Verlag: Berlin, 2007.

(48) Borghese, F.; Denti, P.; Saija, R.; Toscano, G.; Sindoni, O. I. Multiple Electromagnetic Scattering from a Cluster of Spheres. I. Theory. Aerosol Sci. Technol. 1984, 3, 227-235.

(49) Mackowski, D. W.; Mishchenko, M. I. Calculation of the T Matrix and the Scattering Matrix for Ensembles of Spheres. J. Opt. Soc. Am. A 1996, 13, 2266-2278.
(50) Saija, R.; Iatì, M. A.; Borghese, F.; Denti, P.; Aiello, S.; CecchiPestellini, C. Beyond Mie Theory: the Transition Matrix Approach in Interstellar Dust Modeling. Astrophys. J. 2001, 559, 993-1004.

(51) Saija, R.; Iatì, M. A.; Denti, P.; Borghese, F.; Giusto, A.; Sindoni, O. I. Efficient Light-Scattering Calculations for Aggregates of Large Spheres. Appl. Opt. 2003, 42, 2785-2793.

(52) Borghese, F.; Denti, P.; Saija, R; Iatì, M. A.; Sindoni, O. I. Optical Properties of a Dispersion of Anisotropic Particles with NonRandomly Distributed Orientations. The Case of Atmospheric Ice Crystals. J. Quant. Spectrosc. Radiat. Transfer 2001, 70, 237-251.

(53) Iatì, M. A.; Cecchi-Pestellini, C.; Williams, D. A.; Borghese, F.; Denti, P.; Saija, R.; Aiello, S. Porous Interstellar Grains. Mon. Not. R. Astron. Soc. 2001, 322, 749-756.

(54) Yue, G.; Wu, J.; Xiao, Y.; Ye, H.; Lin, J.; Huang, M. Flexible Dye-Sensitized Solar Cell Based on PCBM/P3HT Heterojunction. Chin. Sci. Bull. 2011, 56, 325-330.

(55) Krätschmer, W.; Lamb, L. D.; Fostiropoulos, K.; Huffman, D. R. Solid $\mathrm{C}_{60}$ : A New Form of Carbon. Nature 1990, 347, 354-358.

(56) Hwang, I.; Scholes, G. D. Electronic Energy Transfer and Quantum-Coherence in $\pi$-Conjugated Polymers. Chem. Mater. 2011, 23, 610-620.

(57) Camposeo, A.; Pensack, R. D.; Moffa, M.; Fasano, V.; Altamura, D.; Giannini, C.; Pisignano, D.; Scholes, G. D. Anisotropic Conjugated Polymer Chain Conformation Tailors the Energy Migration in Nanofibers. J. Am. Chem. Soc. 2016, 138, 15497-15505.

(58) Sariciftci, N. C.; Smilowitz, L.; Heeger, A. J.; Wudl, F. Photoinduced Electron Transfer from a Conducting Polymer to Buckminsterfullerene. Science 1992, 258, 1474-1476.

(59) Zhou, R.; Chen, W.; Jiang, X.; Wang, S.; Gong, Q. Enhanced Exciton Migration in Electrospun Poly[2-methoxy-5-(2'-ethylhexyloxy)-1,4-phenylenevinylene]/poly(vinyl pyrrolidone) Nanofibers. Appl. Phys. Lett. 2010, 96, 133309.

(60) Greenfeld, I.; Fezzaa, K.; Rafailovich, M.; Zussman, E. Fast X-ray Phase-Contrast Imaging of Electrospinning Polymer Jets: Measurements of Radius, Velocity, and Concentration. Macromolecules 2012, $45,3616-3626$

(61) Shrotriya, V.; Ouyang, J.; Tseng, R. J.; Li, G.; Yang, Y. Absorption Spectra Modification in Poly(3-hexylthiophene):Methanofullerene Blend Thin Films. Chem. Phys. Lett. 2005, 411, 138-143.

(62) Ruderer, M. A.; Metwalli, E.; Wang, W.; Kaune, G.; Roth, S. V.; Müller-Buschbaum, P. Thin Films of Photoactive Polymer Blends. ChemPhysChem 2009, 10, 664-671.

(63) Fazio, B.; Artoni, P.; Iati, M. A.; D’Andrea, C.; Lo Faro, M. J.; Del Sorbo, S.; Pirotta, S.; Gucciardi, P. G.; Musumeci, P.; Vasi, C. S.; Saija, R.; Galli, M.; Priolo, F.; Irrera, A. Strongly Enhanced Light Trapping in a Two-Dimensional Silicon Nanowire Random Fractal Array. Light: Sci. Appl. 2016, 5, e16062.

(64) Peet, J.; Heeger, A. J.; Bazan, G. C. Plastic" Solar Cells: SelfAssembly of Bulk Heterojunction Nanomaterials by Spontaneous Phase Separation. Acc. Chem. Res. 2009, 42, 1700-1708.

(65) Dang, M. T.; Hirsch, L.; Wantz, G. P3HT:PCBM, Best Seller in Polymer Photovoltaic Research. Adv. Mater. 2011, 23, 3597-3602.

(66) Liu, H.; Edel, J. B.; Bellan, L. M.; Craighead, H. Electrospun Polymer Nanofibers as Subwavelength Optical Waveguides Incorporating Quantum Dots. Small 2006, 2, 495-499.

(67) Persano, L.; Camposeo, A.; Di Benedetto, F.; Stabile, R.; Laera, A. M.; Piscopiello, E.; Tapfer, L.; Pisignano, D. CdS-Polymer Nanocomposites and Light-Emitting Fibers by In Situ Electron-Beam Synthesis and Lithography. Adv. Mater. 2012, 24, 5320-5326.

(68) Ren, S.; Chang, L.-Y.; Lim, S.-K.; Zhao, J.; Smith, M.; Zhao, N.; Bulovic, V.; Bawendi, M.; Gradecak, S. Inorganic-Organic Hybrid Solar Cell: Bridging Quantum Dots to Conjugated Polymer Nanowires. Nano Lett. 2011, 11, 3998-4002.

(69) Fasano, V.; Moffa, M.; Camposeo, A.; Persano, L.; Pisignano, D. Bright Light Emission and Waveguiding in Conjugated Polymer Nanofibers Electrospun from Organic Salt Added Solutions. Macromolecules 2015, 48, 7803-7809.

(70) Manuelli, A.; Persano, L.; Pisignano, D. Flexible Organic FieldEffect Transistors Based on Electrospun Conjugated Polymer 
Nanofibers with High Bending Stability. Org. Electron. 2014, 15,

$1056-1061$. 